\title{
Efficacy of Amphotericin B in a Fat Emulsion for the Treatment of Cryptococcal Meningitis in AIDS Patients
}

\author{
Fernando Góngora Rubio' ${ }^{1}$, Jeferson Rodrigo Zanon², Margarete Teresa Gottardo de Almeida ${ }^{1,3}$ \\ and Delzi Vinha Nunes de Góngora ${ }^{1}$ \\ ${ }^{1}$ Infectious, Parasitary and Dermatologic Diseases Department of São José do Rio Preto Medical School; ${ }^{2}$ Internal Medicine Resident - \\ Hospital de Base - São José do Rio Preto Medical School Fundation; ${ }^{3}$ Microbiology Laboratory of the Infectious, Parasitary and \\ Dermatologic Diseases Department of São José do Rio Preto Medical School; São José do Rio Preto, SP, Brazil
}

\begin{abstract}
Several formulae have been developed in an attempt to reduce the toxicity of amphotericin B (AmB), but their high costs preclude widespread use. The aim of this study was to evaluate the efficacy of amphotericin B in a fat emulsion, i.e. Intralipid (AmB-IL), in 37 AIDS patients with cryptococcal meningitis (CM). We retrospectively reviewed data collected in a non-comparative open study between January 1999 and December 2001. The therapeutic cure was defined as complete resolution or improvement of the clinical symptoms or complete absence or improvement of the mycological alterations of the CSF. The outcomes were evaluated at 2 weeks, induction phase (IP), and at the end of treatment or consolidation phase (CP) with the last available CSF. Prior to the diagnosis of CM, $72 \%$ of patients had had one or more OI and $67.57 \%$ had a concomitant $O I$. The median $\mathrm{CD}_{4}$-cell count was 32 cells $/ \mathrm{mm}^{3}$, the median leukocyte count in the CSF was 29 cells $/ \mathrm{mm}^{3}$ and the median cumulative dose of AmB-IL was 1,200 mg (300-2,500). The therapeutic cure was $57.14 \%$ in the IP and 64.86\% in the CP. During IP, 9 patients died (24.32\%) and 4 (10.81\%) during the $\mathrm{CP}(\mathrm{p}=0.2)$. Thus, the overall mortality rate was $35.14 \%$. AmB-IL, an inexpensive preparation, might be an alternative to conventional AmB. Some questions remain such as its compatibility, stability and level of toxicity. The benefit is especially important in developing countries, where no drugs other than AmB are available to treat systemic fungal infections.
\end{abstract}

Key-Words: Amphotericin B, fat emulsion, cryptococcal meningitis, AIDS patients.

Cryptococcus neoformans is an important pathogen for immunocompromised hosts, in particular for AIDS patients, occurring in $5 \%$ to $10 \%$ of these cases [1]. Ninety percent of infected patients evolve with cryptococcal meningitis. In Brazil, extra-pulmonary cryptococcosis is the eighth most frequent opportunist infection (OI) associated with AIDS [2] and in the USA it is the third most common infection to cause neurological diseases [1], with a mortality rate of between $15 \%$ and $30 \%$, chiefly seen in the first 2 weeks of treatment [3].

Studies performed over the last decade recommend that the first-line treatment of cryptococcal meningitis (CM) in patients with AIDS should be amphotericin B (AmB) at a intravenous dose of 0.7 to $1 \mathrm{mg} / \mathrm{kg}$ daily, combined with 100 $\mathrm{mg} / \mathrm{Kg}$ of 5 -fluorcytosine via oral for 2 weeks, followed by 400 $\mathrm{mg}$ /day of fluconazole for at least 10 weeks to complete the treatment. As a suppressive therapy, fluconazole is utilized at a dose of $200 \mathrm{mg} /$ day until immunological reconstitution [46]. Therapeutic success, when AmB is used at a dose of 0.3 to $0.5 \mathrm{mg} / \mathrm{Kg} /$ day, is $40 \%$, [7] whereas using a dose of $0.7 \mathrm{mg} /$ $\mathrm{Kg} /$ day it is $51 \%$ in respect to the mycological response and clinically 83\% [4]. The administration of AmB is associated

Received on 18 October 2006; revised 17 March 2007.

Address for correspondence: Dr. Fernando Góngora Rubio. Rua Sebastião Bernardino de Souza, 100, - Brejo Alegre. Zip code: 15062-001, São José do Rio Preto - SP, Brazil. Phone: (55 17) 3201:5059; 3238-1171. Phone/Fax: (55 17) 3201-5000- 1582. E-mail: gongora@famerp.br. This project was developed with a Scientific Initiation Grant awarded by the Research Directorate of the Medical School of São José do Rio Preto, in the period from April of 2002 to March of 2003.

The Brazilian Journal of Infectious Diseases 2007;11(2):203-207. (C) 2007 by The Brazilian Journal of Infectious Diseases and Contexto Publishing. All rights reserved. with toxicity, related to the infusion in $70 \%$ to $90 \%$ of patients, and toxicity inherent to the therapy (nephrotoxicity, cardiotoxicity, anemia and hydroelectrolytic abnormalities), with nephrotoxicity being the most significant, seen from $24 \%$ to $80 \%$ of treated patients [8].

To reduce the toxicity, several studies have suggested new formulations of AmB (lipophilic drug) in lipid transporters (small unilamellar liposomes-AmBisome; colloidal dispersion - ABCD or amphocil and lipid complexes - ABLC or abelcet); but, they are little used due to their high cost $[9,10]$. Thus, an alternative system based on $\mathrm{AmB}$ in the fat emulsion intralipid (AmB-IL), clinically used for parenteral nutrition, has given a reduction in the adverse effects during administration and the nephrotoxicity of AmB in neutropenic and critically ill patients, as well as providing a good therapeutic efficacy [10-14].

In respect to the treatment of cryptococcal meningitis in AIDS patients, AmB has a similar efficiency to conventional deoxycholate AmB (D-AmB) [15-17], with a reduction in the toxicity related to infusion $[16,17]$, but without substantial benefits in terms of protection against renal toxicity, mainly with higher doses of AmB [15,17,18].

The aim of this study was to evaluate the therapeutic efficacy of AmB-IL use in AIDS patients with CM.

\section{Materials and methods}

Study Institution

Hospital de Base is a 420-bed general teaching hospital linked to the Medical School in São José do Rio Preto, São Paulo, Brazil. It has an AIDS unit for 25 hospitalized patients and attends approximately 900 patients per month in an STD/ AIDS outpatients clinic. 


\section{Study Design}

AIDS patients with CM, diagnosed during the period from January $18^{\text {th }} 1999$ to December $22^{\text {nd }} 2001$, were retrospectively studied through data collection from their patient report cards.

AmB was administered in the following form: doses of 0.7 $\mathrm{mg} / \mathrm{kg} /$ day up to a maximum of $50 \mathrm{mg}$ were mixed with $10 \%$ fat emulsion (Intralipid) and continuously infused over a 1-hour period; the dose prior to the test was not considered. Corticosteroid (50 mg hydrocortisone) or antipyretic (500 mg dipyrone) administration was made depending on the side effects and prior to $\mathrm{AmB}$ administration, volume expansion with $500 \mathrm{~mL}$ of $0.9 \%$ saline solution was performed over 30 to 60 minutes.

The therapy employed was an induction phase (IP) using a daily administration of AmB for 15 days as in aforementioned doses. Following this there was a consolidation phase (CP) when $A m B$ was administered 3 times per week until a complete or partial cure was achieved or death, characterizing the end of the treatment (ET), according to the obtained final clinical follow up.

Patients who took at least $700 \mathrm{mg}$ of the drug, which normally happened within the first 2 weeks of treatment, that is the IP, were considered in the analysis of the efficacy of the drug.

The intervals of data collection were the day of diagnosis, the initial day of treatment (day zero); the last day of the IP after an accumulated dose of $700 \mathrm{mg}$ (D 700); day of the last dose in the CP. In cases that 3 was no data in respect to the cerebrospinal fluid (CSF) in the CP, the last CSF collected was considered as the end of treatment for the evaluation of efficacy. For the last day of the IP, the maximum interval of data collection and of examinations was 3 days before or after the expected date; for the last day of the $\mathrm{CP}$, the interval was 7 days before or after the stipulated date.

\section{Population}

Inclusion of patients was made using the following criteria: age greater than or equal to 13 years old; anti-HIV positive and positive China ink or latex tests of the CSF or had a positive culture for cryptococcosis along with clinical signs. Patients with positive latex tests without clinical signs were considered cases when the treatment was instituted by an assistant physician. The first episode of CM in the patients was considered for the study.

The following characteristics were used as exclusion criteria: patients with serum creatinine levels higher than 2.5 $\mathrm{mg} / \mathrm{dL}$; incomplete follow-ups; patients who were administered AmB diluted in 5\% dextrose or other antifungal agents associated or not with AmB for more than 48 hours immediately before or during the hospital stay.

Epidemiological data were collected including epidemiology of HIV (sexually transmitted, use of injected drugs and blood transfusions), prior OI and OI concomitant with the current diagnosis of CM. Clinical and laboratorial variables related to toxicity were collected: trembling, cold sweats, sudoresis, fever (axillary temperature greater than $37.8^{\circ} \mathrm{C}$ ), arterial hypotension (blood pressure less than $90 \times 60$ $\mathrm{mmHg}$ ), tachycardia (heart frequency greater than $100 \mathrm{bpm}$ ), phlebitis at the infusion site, serum potassium of less than $3.5 \mathrm{mEq} / \mathrm{L}$, nephrotoxicity when the serum creatinine increased by $0.5 \mathrm{mg} / \mathrm{dL}$ from the initial sampling and in cases in which the initial creatinine was not measured, a value greater than $1.4 \mathrm{mg} / \mathrm{dL}$.

\section{Analysis of Efficacy}

To analyze the efficacy, clinical and laboratorial (CSF) data were evaluated in parallel. The following symptoms were assessed: cephalea; vomiting; behavioral disorders defined as psychomotor depression or agitation; stiff neck; epileptic crises; decreased levels of awareness and coma. Clinical definitions of the efficacy were as follows:

Clinical cure - complete clinical resolution of symptoms in the $\mathrm{CP}$ or with the last administered dose.

Clinical improvement - partial solution of symptoms (at least 2).

Clinical failure - appearance of new symptoms in the CP. Without clinical change - continuation of the symptoms.

From the China ink test and culture of the CSF the presence and amount of cryptococcal were assessed. Laboratorial definitions of the efficacy were:

Laboratorial cure - absence of cryptococcal in the CSF and at least 1 negative culture for cryptococcal during the treatment or at the last administered dose.

Laboratorial improvement - a reduction in the number of cryptococcal in relation to the CSF at admission or if cryptococcal were not identified in the control CSF, a negative culture for cryptococcal.

Laboratorial failure - existence of cryptococcal in the CSF or an increase in the number of cryptococcal during the treatment or at the last administered dose.

Without laboratorial change - control CSF did not present significant changes in relation to CSF at admission in respect to the China Ink, latex tests, number of cryptococcal, cryptococcal gemulation and culture.

Definitions of the efficacy of the treatment:

Therapeutic cure - one of the following: 1) clinical and laboratorial cure; 2) clinical and laboratorial improvement; 3) clinical improvement and laboratorial cure or clinical cure and laboratorial improvement; 4) clinical improvement or cure without laboratorial change; 5) laboratorial improvement or cure without clinical change.

Therapeutic failure - one of the following: 1) clinical and laboratorial failure; 2) clinical or laboratorial failure and clinical or laboratorial improvement.

Without change - was defined as without clinical and laboratorial changes.

The following situations were not assessed due to the lack of data: 1) patients initially included who did not complete at least $700 \mathrm{mg}$ of treatment (IP), except for patients who died in this phase; 2) when at least the results of 2 CSF tests were 
not found in the patient's records or 3) when 2 CSF tests were made with the last showing a level of less than $700 \mathrm{mg}$.

\section{Statistic Analysis}

The Chi-square and Fischer exact tests were utilized for the analysis of the outcomes and the level of rejection of the null hypothesis was set at 0.05 or $5 \%$ (alpha less than or equal to 0.05 ).

\section{Results}

Population

During the period from January $18^{\text {th }} 1999$ to December $22^{\text {nd }}$ 2001, 58 AIDS patients diagnosed with cryptococcal meningitis (CM) were studied. Table 1 demonstrates that, of these, 21 patients (36.20\%) were not included in the study to evaluate the efficacy for the following reasons: 5 (8.62\%) had incomplete data due to misplaced patient report cards; 2 patients $(3.45 \%)$ were excluded, one of them due to prior fluconazole use and the other for AmB use diluted in 5\% dextrose. Fourteen patients (24.13\%) had incomplete data, 7 (12.07\%) of whom used fluconazole before the accumulated dose of $700 \mathrm{mg}$ of AmB, 2 patients (3.44\%) abandoned the treatment, 3 patients $(5.17 \%)$ transferred treatment to another service and 2 patients (3.44\%) because there was no control CSF test. Thirty-seven patients (63.80\%) were included in the study of the efficacy with 8 patients (13.80\%) dying before the end of the IP and 29 (50\%) were evaluated both at the end of the IP and in the CP (Table 1).

The distributions of the epidemiological, clinical and laboratorial data of the 37 cases were as follows: the median age was 33 years (ranging from 20 to 60 years) with $83.78 \%$ men patients. The transmission of HIV in this population was: sexual in 20/37 (54.05\%), drug use in 3/37 (8.11\%), sexual and drug use in $3 / 37$ (8.11\%) and unknown cause 11/37 (29.73\%). The distribution of the number of OI related to the CM diagnosis was: 2 or more previous OI in 20/37 (54.05\%), only 1 previous OI in 7/37 (18.92\%); 2 or more concomitant OI in $8 / 37$ (21.62\%) and only 1 concomitant OI in $17 / 37$ (45.95\%).

In the first CSF examination the following data were observed: a median of 29 leukocytes (ranging from 1 to 430) with a mean of 64.57 (standard deviation \pm 93.3 ); median number of cryptococcal was 185 (varying from 0 to 4,480 ) with a mean of 632.11 (standard deviation $\pm 1,174.9$ ). The mean CD count was 54.77 cells (standard deviation \pm 52.74 ) with a median of 32 (variation from 1-191). The accumulative dose of AmB varied from 300 to 2,500 mg with a median of $1200 \mathrm{mg}$.

\section{Analysis of the Efficacy}

In respect to the evaluation of the therapeutic response to AmB in fat emulsion in 37 AIDS patients with CM during the study period, 8/37 died before completing an accumulated dose of $700 \mathrm{mg}$ in the IP. Even though these patients did not make the control CSF test after a dose of $700 \mathrm{mg}$ of AmB, they were included in the analysis of the therapeutic response and considered a failure to the treatment as the CM was the cause of death on the death certificate (Table 1).

From the total of patients studied, 28 were evaluated in the IP. Eleven, 11/28 (39.28\%) failing treatment, 16/28 (57.14\%) cured and 1/28 (3.57\%) did not change patients (Table 2).

Twenty-three patients were studied in the CP, with 4/23 (17.39\%) failing treatment and 19/23 (82.61\%) cured patients (Table 2).

Of the patients studied, only 29 patients were evaluated at the ET (18/29 at the last dose, with 3 patients with an accumulated dose of $700 \mathrm{mg}$, and $11 / 29$ patients with the last dose and CSF concomitantly) and 8 deaths in the IP presenting with the following results: 24/37 (64.86\%) were cured and 13/ 37 (35.14\%) failing treatment (Table 2).

\section{Deaths}

During the study period, 13 patients died presenting an overall mortality rate of 35.14\%; with 9/37 (24.32\%) dying in the IP and 4/37 (10.81\%) in the CP, giving a higher mortality rate in the first phase compared to the second, but without significant difference $(\mathrm{p}=0.2)$.

\section{Toxicity of the Drug}

In respect to the toxicity of $\mathrm{AmB}$ in the 37 evaluated patients, 19/37 (51.35\%) presented some adverse effects related to the infusion of the drug, which included fever in 12/ 37 (32.43\%), tachycardia in 11/37 (29.73\%), arterial hypotension in $8 / 37$ (21.62\%), trembling in 5/37 (13.51\%), phlebitis in 3/37 (8.11\%), hydrorrhea in 2/37 (5.41\%) and cold sweat in 1/37 (2.70\%). Effects not related to the infusion included hypokalemia in 20/37 (54.05\%) and nephrotoxicity in 22/37 (59.46\%).

\section{Discussion}

The treatment of CM in AIDS patients has already been defined in the literature as utilizing AmB and 5-fluorocytosine for 2 weeks, followed by fluconazole [4]. However, few studies have evaluated the therapeutic efficacy of AmB-IL [15-18] and only 1 open controlled and randomized study compared AmB with AmB-IL, employing doses of $0.7 \mathrm{mg} / \mathrm{kg} /$ day and 1 $\mathrm{mg} / \mathrm{kg} /$ day respectively, but without 5-fluorocytosine [17]. Our open non-comparative study, retrospectively evaluates the use of AmB-IL without 5-fluorocytosine at a dose of $0.7 \mathrm{mg} /$ $\mathrm{Kg} /$ day with the administration of a median of 1,200 $\mathrm{mg}$ of AmB-IL. In this study, patients who were treated for at least 2 weeks (IP) were evaluated and, for patients who continued until a cure or a change of treatment using other antifungal agents, the ET was defined as the last dose of AmB-IL or the last dose associated to the last available CSF test.

Of 37 cases studied, 31 patients presented a median CD count of 32 cells $/ \mathrm{mL}$, demonstrating the important degree of immunosuppression of this population. In this study, patients in coma or those with concomitant opportunist infections were not excluded, different of the study of Van der Horst [4], illustrating the serious state of the studied patients. 
Table 1. Total of 58 cases of AIDS patients diagnosed with cryptococcal meningitis in the period from 18/01/1999 to 22/12/2001

\begin{tabular}{lrr}
\hline & $\mathbf{N}$ & $\mathbf{\%}$ \\
\hline Patients not included in the study & & \\
Misplaced report cards & 05 & 08.62 \\
Exclusions & 02 & 03.45 \\
Incomplete data & 14 & 24.13 \\
Subtotal & 21 & 36.20 \\
Patients included in the study & & \\
Deaths before completing the induction phase (D 700 mg) & 08 & 13.80 \\
Evaluation at a cumulated dose of 700 mg or during the consolidation phase & 29 & 50.00 \\
Subtotal & 37 & 63.80 \\
Total & 58 & 100.00 \\
\hline
\end{tabular}

Table 2. Evaluation of the therapeutic response of Amphotericin B in fat emulsion of 37 AIDS patients with cryptococcal meningitis in the period of 18/01/1999 to 22/12/2001

\begin{tabular}{lccccccc}
\hline Population & \multirow{2}{*}{} & \multicolumn{5}{c}{ Therapeutic response } \\
\cline { 3 - 9 } & & \multicolumn{2}{c}{ Failure } & \multicolumn{2}{c}{ Cure } & \multicolumn{2}{c}{ Without change } \\
\cline { 3 - 9 } & & $\mathbf{N}^{\mathbf{0}}$ & $\mathbf{0}$ & $\mathbf{N}^{\mathbf{0}}$ & $\mathbf{\%}$ & $\mathbf{N}^{\mathbf{0}}$ & $\mathbf{\%}$ \\
\hline Induction phase & $28 / 37$ & $11 / 28$ & 39.28 & $16 / 28$ & 57.14 & $1 / 28$ & 3,57 \\
Consolidation phase & $23 / 37$ & $4 / 23$ & 17.39 & $19 / 23$ & 82.61 & 00 & 00 \\
End of treatment & $37 / 37$ & $13 / 37$ & 35.14 & $24 / 37$ & 64.86 & 00 & 00 \\
\hline
\end{tabular}

Concerning the evaluation of efficacy criteria, we utilized the presence and the number and variation of cryptococcal in the CSF as well as from the culture to define therapeutic success because of the availability of a laboratory specialized in assessing the level of cryptococcal.

We obtained a therapeutic cure rate of $57.14 \%$ during the IP, lower than the best published rate using D-AmB (83\%), which was in a controlled multicenter, randomized and doubleblind clinical trial, with patients in coma and those with multiple organ dysfunction being excluded, justifying the excellent results of the trial. In the same study, the clearing of the CSF occurred in $51 \%$ of the patients who used D-AmB [4]. The majority of the studies measured the global clinical response and do not separately consider the first 2 weeks of treatment to evaluate the efficacy. The Van der Horst study did not perform an overall analysis of the first 2 weeks, which was possible in our work as we established the definitions with clinical and laboratorial criteria. However, there are few published data to compare our response directly with our defining criteria. Nevertheless, our response gave an intermediate result when compared with the response of Van der Host. When we evaluated the overall results at the ET we had a cure of $64.86 \%$, a response similar to or better than other studies that evaluated AmB-IL: $70 \%$ complete or partial solution of the symptoms [15], 73.8\% of cure or clinical improvement, $69 \%$ of mycological cure [17], 55.56\% with cure or clinical improvement and $55.56 \%$ with mycological response [18]. Compared to Saag et al., who also considered the therapeutic success with analysis of both the clinical improvement and clearing of the CSF, whose result was $40 \%$
[7], we obtained a better response. These authors, however, used no more than $0.5 \mathrm{mg} / \mathrm{kg} /$ day of D-AmB, which may explain the worse response. The analysis of the efficacy, as can be seen, depends very much on the type of patients included in the study and those excluded, on the pre- or post-HAART, on the study type, on the definitions employed, on the different drugs and doses used and on the different periods assessed. Thus, it is difficult to find a study that we can use as a reference to compare our results. However, we confirmed that our overall response is similar to published results. We did not observe any association between therapeutic success and the presence of OI or the CD4 count (p-value $>0.05$ ). We confirmed, however, a relationship between higher doses of AmB-IL (more than $1200 \mathrm{mg})$ and therapeutic success $(\mathrm{p}=0.004)$ as was expected.

When we studied mortality, we found published rates of 5.5\% [4] and 14\% [7] and variations between 15\% and 30\% [3], with deaths occurring mainly within the first 2 weeks of treatment with $\mathrm{D}-\mathrm{AmB}$ in the last case. The overall mortality rates of patients who used AmB-IL found in the literature are $30 \%$ [15], 22.22\% [18] and 16.7\% [17], similar to our results (35.14\%), with the majority of the deaths (24.32\%) occurring during the first 2 weeks of treatment, but with a non-significant statistical difference with the deaths that occurred in the CP (p-value $=0.2$ ). Due to the retrospective nature of our work, it was not possible to perform a detailed evaluation of the state of the patients, but as most deaths occurred during the IP, this suggests that the patients were severely ill.

In conclusion, we verified that the therapeutic response evidenced in our study using AmB-IL was similar in efficacy 
to results previously described, thereby contributing to the few available published data. Thus, AmB-IL is a cheap formulation that may provide an alternative in the treatment of cryptococcal meningitis and systemic fungal diseases, especially in poorer countries. Some questions still require to be better documented such as its compatibility, stability and the reduced toxicity of this emulsion.

\section{References}

1. Powderly W.G. Cryptococcal meningitis and AIDS. Clin Infect Dis 1993; 17: 837-42.

2. Boletim epidemiológico AIDS. Ano XIV no 01 - semana epidemiológica $1^{\mathrm{a}}$ a $13^{\mathrm{a}} / \mathrm{jan}$.fev.mar - 2001.

3. Dismukes W.E. Management of cryptococcosis. Clin Infect Dis 1993;17(Suppl 2):S507-12.

4. Van der Horst C.M., Saag M.S., Cloud G.A., et al. Treatment of criptococcal meningitis associated with the acquired immunodeficiency syndrome. $\mathrm{N}$ Engl $\mathrm{J}$ Med 1997;337(21):1557-8.

5. Powderly W.G. Current approach to the acute management of cryptococcal infections. Journal of Infection. 2000;41:18-22.

6. Saag M.S., Graybill R.J., Larsen R.A., et al. Practice guidelines for the management of cryptococcal disease. Clin Infect Dis 2000;30:710-8.

7. Saag M.S., Powderly W.G., Cloud G.A., et al. Comparison of amphotericin B with fluconazole in the treatment of acute AIDS - associated cryptococcal meningitis. N Engl J Med 1992;326:83-9.

8. Gallis H.A., Drew R.H., Pickard W. Amphotericin B: 30 years of clinical experience. Rev Infect Dis 1990;12:308-29.

9. Craven P.C., Gremillion D.H. Risk factors of ventricular fibrillation during rapid amphotericin B infusion. Antimicrob Agents Chemother 1985;27:868-71.
10. Egito E.S.T., Araújo I.B., Damasceno B.P.G.L., Price J.C. Amphotericin B/emulsion admixture interactions: an approach concerning the reduction of amphotericin B toxicity. Journal of Pharmaceutical Sciences 2002;91:2354-66.

11. Nucci M., Loureiro M., Silveira F., et al. Comparision of the toxicity of amphotericin B in fat emulsion in a randomized trial with cancer pacients. Antimicrobial Agents and Chemotherapy 1999;43:1445-8.

12. Sorkine P., Nagar H., Weinbroum A., et al. Administration of amphotericin B in lipid emulsion decreases nephrotoxicity: results of a prospective, randomized, controlled study in critically ill pacients. Crit Care Med 1996;24:1311-15.

13. Caillot D., Casanovas O., Solary E., et al. Efficacy and tolerance of an amphotericin B lipid (Intralipid) emulsion in the treatment of candidaemia in neutropenic patients. J Antimicrob Chemother 1993;31:161-9.

14. Moreau P., Milpied N., Fayette N., et al. Reduced renal toxicity and improved clinical tolerance of amphotericin B mixed with intralipid compared with conventional amphotericin B in neutropenic patients. J Antimicrob Chemother 1992;30:535-41.

15. Torre D., Banfi G., Tambini R., et al. A retrospective study on the efficacy and safety of amphotericin B in a lipid emulsion for the treatment of cryptococcal meningitis in AIDS patients. Journal of Infection 1998;37:36-8.

16. Manfredi R., Chiodo F. Case-control study of amphotericin B in a triglyceride fat emulsion versus conventional amphotericin B in patients with AIDS. Pharmacotherapy 1998;18(5):1087-92.

17. Joly V., Pierre A., Ndayiragide A., et al. Randomized comparison of amphotericin B deoxycholate dissolved in dextrose or intralipid for the treatment of AIDS-associated cryptococcal meningitis. Clin Infect Dis 1996;23:556-62.

18. Joly V., Geoffray C., Reynes J., et al. Amphotericin B in a lipid emulsion for the treatment of cryptococcal meningitis in AIDS patients. Journal of Antimicrobial Chemotherapy 1996;38:117-26. 